\title{
Potencialidades y desafíos de la extensión universitaria para el trabajo en derechos humanos, género y discapacidad
}

\author{
María Lilián González ${ }^{1}$ Y Tania da Rosa ${ }^{2}$
}

Recibido: 23/03/2021; Aceptado: 16/06/2021

DOI: https://doi.org/10.37125/ISR.7.1.3

\section{Resumen}

El artículo plantea la potencialidad de la extensión universitaria como campo de acción para llevar adelante los distintos roles de la Universidad en relación con los derechos humanos. Reflexiona sobre la convergencia y retroalimentación de objetivos y metodologías de la extensión y la educación en derechos humanos. Enfatiza la necesidad de transversalizar la temática de la discapacidad en la extensión y en todo el quehacer de la Universidad, profundizando para ello el análisis sobre los entornos y su habilitación a la diversidad. El artículo subraya la importancia de integrar a las prácticas de extensión en derechos humanos la perspectiva de la interseccionalidad como herramienta indispensable para el análisis, el trabajo y la construcción conjunta con los colectivos sociales. A partir de este marco conceptual las autoras presentan una experiencia de extensión desarrollada desde el Área de Derechos Humanos del Servicio Central de Extensión de la Universidad de la República con el Colectivo Mujeres y Discapacidad en el contexto de pandemia.

Palabras clave: género, discapacidad, derechos humanos.

\section{Un posible punto de partida:}

\section{extensión, derechos humanos y discapacidad}

La temática discapacidad y derechos humanos es uno de los ejes de trabajo del Área de Derechos Humanos del Servicio Central de Extensión y Actividades en el Medio (SCEAm) de la Universidad de la República (Udelar).

Desde este espacio universitario, la discapacidad es vista como campo amplio de abordaje, de estudio y de intervención. Existen distintas definiciones asociadas al concepto de discapacidad, concepto que ha evolucionado históricamente y el cual se ha

1 Servicio Central de Extensión y Actividades en el Medio (sCEAM), Universidad de la República (Udelar). marialiliangonzalez@gmail.com

2 SCEAM, Udelar. tania.darosa@cseam.udelar.edu.uy 
asumido que se encuentra en constante reformulación. La Convención Internacional de los Derechos de las Personas con Discapacidad (CPDP)3 (Organización de las Naciones Unidas [ONU], 2006) posiciona esta definición en la relación entre las especificidades de la persona y el entorno que habita. Cuando esa relación es negativa es que se produce la discapacidad, dotando así de movimiento un concepto que históricamente era estático y centrado en la persona.

El hecho de pensar y abordar el tema de la discapacidad desde esta concepción de interrelación, obliga a pensar en los entornos y su habilitación a la diversidad, de cuerpos, de visiones, de escuchas, de desplazamientos, de comprensión. Implica también, abordar las resistencias, los miedos que se despiertan en el encuentro con lo desconocido. Desconocido por ausencia de espacios públicos compartidos, de un tema que está estrictamente vinculado al ámbito de lo privado, la órbita de lo familiar.

El artículo 8 de la CDPD (ONU, 2006) se refiere a la toma de conciencia sobre los derechos de las personas con discapacidad y obliga a los Estados parte a promover una cultura de información, sensibilización y formación sobre estos en los más diversos ámbitos.

La Udelar, como principal institución de educación terciaria del Uruguay, adquiere compromisos múltiples en este tema: la formación de profesionales con un enfoque de derechos humanos en la temática, la contribución a la producción de conocimiento sobre este campo de problemas y la construcción de saberes de manera integral e interinstitucional. Asimismo y en consonancia con el artículo 2 de la Ley Orgánica (1958), le incumbe a la Universidad contribuir a la expansión de una cultura de respeto de los derechos humanos en su integralidad y la defensa de estos. ${ }^{4}$

Todo lo anterior nos lleva a destacar la importancia de incorporar y expandir transversalmente la perspectiva de derechos humanos a todas las funciones universitarias, apostando a la integralidad como marco teórico y metodológico para reforzar el compromiso de la Universidad con la sociedad.

3 La Convención adoptada en el ámbito de la onU en 2006, fue ratificada por Uruguay en 2008. El artículo 1 indica: «Las personas con discapacidad incluyen a aquellas que tengan deficiencias físicas, mentales, intelectuales o sensoriales a largo plazo que, al interactuar con diversas barreras, puedan impedir su participación plena y efectiva en la sociedad, en igualdad de condiciones con las demás». La CPDP supuso un cambio de paradigma en los enfoques de la discapacidad explicitando y promoviendo el reconocimiento de las personas en situación de discapacidad como sujetos de derechos humanos; considera que las barreras de la sociedad a que se enfrentan son los principales obstáculos para el pleno disfrute de los derechos humanos.

4 Ley Orgánica de la Universidad de la República, Ley n. ${ }^{\circ}$ 12.549: «Art. 2. Fines de la Universidad. La Universidad tendrá a su cargo la enseñanza pública superior en todos los planos de la cultura, la enseñanza artística, la habilitación para el ejercicio de las profesiones científicas y el ejercicio de las demás funciones que la ley le encomiende. Le incumbe, asimismo, a través de todos sus órganos, en sus respectivas competencias, acrecentar, difundir y defender la cultura; impulsar y proteger la investigación científica y las actividades artísticas y contribuir al estudio de los problemas de interés general y propender a su comprensión pública; defender los valores morales y los principios de justicia, libertad, bienestar social, los derechos de la persona humana y la forma democrático-republicana de gobierno». 
Posicionarse desde la perspectivas de derechos humanos, afirma Fernando Willat (2011), involucra necesariamente tres dimensiones: ética, política y jurídica. El autor sintetiza de la siguiente manera los alcances de construir políticamente en clave de derechos humanos:

El proyecto político del enfoque de derechos humanos es por lo tanto un proyecto de empoderamiento de los sujetos para que asuman la conducción autónoma de sus propios destinos y para que los otros también puedan empoderarse y asumirla. Es un proyecto que busca la redistribución del poder y la eliminación de las relaciones de dominación (Willat, 2011, p. 22).

En consonancia con lo desarrollado hasta aquí entendemos a la extensión universitaria como un campo de acción indispensable y una oportunidad para llevar a la práctica los diversos roles de la Universidad en relación con los derechos humanos. Así lo pauta la vocación transformadora de la extensión hacia el demos universitario y hacia la sociedad en su conjunto mediante el reconocimiento de los actores sociales como protagonistas de tales procesos y la apuesta a la construcción conjunta de saberes.

Asimismo, asumiendo el desafío que plantea Eloísa Bordoli (2010)5 de pensar la «potencialidad educativa» de la extensión en relación con los actores universitarios y no universitarios, quisiéramos también referirnos a la extensión universitaria como una oportunidad y una práctica de y para la «educación en, por y para los derechos humanos».

La Declaración de las onU sobre educación y formación en materia de derechos humanos (2011) plantea:

La educación y la formación en materia de derechos humanos engloban:

a. La educación sobre los derechos humanos, que incluye facilitar el conocimiento y la comprensión de las normas y principios de derechos humanos, los valores que los sostienen y los mecanismos que los protegen;

b. La educación por medio de los derechos humanos, que incluye aprender y enseñar respetando los derechos de los educadores y los educandos;

c. La educación para los derechos humanos, que incluye facultar a las personas para que disfruten de sus derechos y los ejerzan, y respeten y defiendan los de los demás (onU, 2011, art. 2.2).

5 Según reflexiona la autora, «... la extensión adquiere una potencialidad educativa para docentes, estudiantes y agentes comunitarios en tanto reafirme canales de participación y búsqueda conjunta de soluciones efectivas». Desde este enfoque, «el meollo está en la necesidad de democratizar, poner a disposición del conjunto social aquellos conocimientos — precarios y limitados- que habiliten nuevos procesos de creación de saberes y que se hallen en relación con las necesidades del medio» (Bordoli, 2010, p. 18). 
Por eso, como plantea Rosa Mujica (2002), educar en derechos humanos no consiste solamente en compartir o transmitir conocimientos sobre los derechos humanos, sus fundamentos, normas y principios, sino que la metodología y la actitud de quien asume este desafío resultan componentes intrínsecos e imprescindibles. Una metodología como refiere la autora «que parta de la realidad» de las personas con las que trabajamos (Mujica, 2002, p. 354) y que promueva el respeto a la dignidad de las personas y valore su diversidad.

No es propósito específico de este trabajo reflexionar sobre la importancia de la educación en derechos humanos en la Universidad. Al respecto simplemente nos limitaremos a reafirmar la vigencia de la afirmación de Ana María Rodino (2014):

Las universidades - todas- deberían hacerse responsables de educar en derechos humanos dentro de las disciplinas que imparten - todas-. Porque su estudio tiene impacto en varios niveles: (i) en la formación integral de los estudiantes; (ii) en el desempeño crítico y socialmente responsable de cualquier profesional; (iii) en el avance del conocimiento disciplinario y social; y (iv) en el progreso de los derechos humanos en la comunidad donde la universidad se inserta (Rodino, 2014, p. 134).

Como se desprende de las citas anteriores, el trabajo con la comunidad y el encuentro de saberes universidad-comunidad, aspectos sustantivos de las prácticas extensionistas, lo son también del por y para de la educación en derechos humanos, y ofrecen un marco teórico y metodológico para la redistribución del poder y el empoderamiento. Desde esta perspectiva podría sostenerse que la extensión y la educación en derechos humanos se complementan y se influyen mutuamente.

Por otro lado, ubicar las prácticas e intervenciones extensionistas desde el paradigma de los derechos humanos conlleva reconocer y afirmar como características centrales de tales derechos, su universalidad, indivisibilidad, interdependencia e interrelación. Implica también, reafirmar y reivindicar el reconocimiento y desarrollo de los derechos humanos como el producto de las sostenidas luchas sociales por superar las atrocidades que ha enfrentado ( $y$ enfrenta) la humanidad, y la desigualdad a la que han sido y son sometidos diversos grupos. Supone, por tanto, subrayar el rol activo de la Universidad en relación con la promoción de una cultura de respeto y plena vigencia de los derechos humanos bajo los principios de igualdad y no discriminación. Desde esta perspectiva, la extensión universitaria contribuye a la generación de condiciones para el empoderamiento - particularmente de los grupos más postergados de la sociedad - para la exigibilidad de la plena efectividad de sus derechos y la transformación social. Nuevamente podemos afirmar que los objetivos de la extensión y de la educación en derechos humanos se retroalimentan. 


\section{La discapacidad como campo de trabajo para la extensión universitaria}

El Área de Derechos Humanos del sceam de la Universidad de la República surge del convencimiento de que el enfoque de derechos humanos debe transversalizar las prácticas universitarias. Entre sus cometidos se encuentra "promover una cultura integral de respeto, promoción y plena vigencia de los derechos humanos, en su concepción de un todo interdependiente e indivisible (derechos civiles, políticos, económicos, sociales y culturales» (Servicio Central de Extensión y Actividades en el Medio [SCEAM], 2019). Este cometido solo es posible produciendo sinergia e interacción constante con las instituciones públicas y privadas, así como con distintos colectivos de la sociedad civil que se ocupan de los derechos humanos, por medio del trabajo conjunto con los servicios universitarios.

En el diseño y desarrollo de sus prácticas integrales el Área prioriza temáticamente a aquellos colectivos en mayor situación de vulnerabilidad de sus derechos, a saber: mujeres, personas en situación de discapacidad, población migrante, personas en situación de calle, víctimas de terrorismo de Estado y personas en situación de privación de libertad. Si bien este ejercicio categórico y de delimitación de las distintas líneas de trabajo en las que el Área basa su labor es necesario para su organización, existe plena conciencia de que cada uno de estos temas tienen distintos niveles de cruces e intersecciones.

Asimismo, se reconoce que los posibles cruces temáticos y variables de identidad, no podrían ser analizados sin tener en cuenta el momento histórico y la coyuntura en la que se realiza dicho análisis. Retomamos aquí los aportes de Javiera Cubillos (2015), sobre el concepto de interseccionalidad política, considerándola como aquella que: "permite entender cómo las estrategias políticas que solo se centran en una dimensión de desigualdad marginan de sus agendas a aquellos sujetos o grupos cuya situación de exclusión responde a la imbricación de diversos sistemas de opresión» (Cubillos, 2015, p. 122).

Partiendo de esta afirmación y adentrándonos en el tema específico que atañe a la presente publicación, esta conciencia de interseccionalidad política se puede materializar en el cruce de género, violencia y discapacidad.

\section{Intersección género y discapacidad}

La perspectiva de la interseccionalidad en tanto marco conceptual y categoría de análisis, permite comprender y es sensible, a cómo interactúan diversos factores en la desigualdad y la discriminación estructural que afecta a las mujeres.

Mediante la Observación General número 3 sobre las mujeres y las niñas con discapacidad el Comité sobre los Derechos de las Personas con Discapacidad [CRPD, por 
sus siglas en inglés] $]^{6}$ examinó cómo interactúan el género y la discapacidad (oNU, CRPD, 2016).

$\mathrm{Al}$ respecto, este órgano experto que supervisa la implementación de la CPDP analizó que

... las leyes y políticas internacionales y nacionales sobre la discapacidad han desatendido históricamente los aspectos relacionados con las mujeres y las niñas con discapacidad. A su vez, las leyes y las políticas relativas a la mujer tradicionalmente han hecho caso omiso de la discapacidad. Esta invisibilidad ha perpetuado una situación en la que existen formas múltiples e interseccionales de discriminación contra las mujeres y las niñas con discapacidad. Las mujeres con discapacidad a menudo son objeto de discriminación por motivos de género o discapacidad (ONU, CRPD, 2016, párr. 3).

El comité desarrolló el alcance del concepto de discriminación interseccional indicando que el mismo refiere a una situación en la que varios motivos - la edad, la discapacidad, la identidad de género y la orientación sexual, entre otros- «interactúan al mismo tiempo de forma que son inseparables» (ONU, CRPD, 2016, párr. 4-5). En palabras del Comité:

... el concepto de discriminación interseccional reconoce que las personas no sufren discriminación como miembros de un grupo homogéneo, sino como individuos con identidades, condiciones y circunstancias vitales multidimensionales (ONU, CRPD, 2016, párr. 16).

En ese mismo orden de ideas el órgano experto estableció que las mujeres en situación de discapacidad - que no constituyen un grupo homogéneo- se ven enfrentadas a estereotipos nocivos en base a los cuales experimentan formas estructurales o sistémicas de discriminación. También observó que la intersección género y discapacidad determina formas de discriminación indirectas bajo prácticas aparentemente neutras, pero que afectan de manera desproporcionadamente negativa en las mujeres en situación de discapacidad al no incorporar precisamente la perspectiva de la interseccionalidad.

El Preámbulo de la CPDP reconoce la diversidad de personas con discapacidad y enfatiza: «... la necesidad de incorporar una perspectiva de género en todas las actividades destinadas a promover el pleno goce de los derechos humanos y las libertades fundamentales para las personas con discapacidad» (ONU, 2006, Preámbulo, lit. s).

Asimismo, el artículo 6 de la Convención reconoce que las mujeres en situación de discapacidad están sujetas a múltiples formas de discriminación e impone a los Estados que han ratificado este instrumento, adoptar medidas para asegurar «que

6 El Comité de los Derechos de las Personas con Discapacidad es el órgano integrado por expertos y expertas independientes que supervisa la implementación de la Convención. Todos los Estados parte deben presentar informes periódicos al CRPD, que examina cada informe y emite recomendaciones al Estado en forma de observaciones finales. El CRPD también emite observaciones generales, mediante las cuales precisan o interpretan el alcance concreto de la Convención desarrollando las obligaciones específicas derivadas para todos los Estados que la han ratificado, a fin de garantizar de forma plena y efectiva los derechos reconocidos. 
puedan disfrutar plenamente y en igualdad de condiciones de todos los derechos humanos y libertades fundamentales» (ONU, 2006, art. 6).

No está demás afirmar que este enfoque explicitado por la CPDP sobre los Derechos de las Personas con Discapacidad vino a reforzar y complementar el mandato derivado de los instrumentos del derecho internacional de los derechos humanos que la precedieron, en particular y sin dudas de la Convención sobre la eliminación de todas las formas de discriminación de la mujer (ONU, CEDAW, 1979).

Por tanto, y en función de los antecedentes expuestos, existe una obligación jurídica en cabeza de los Estados de adoptar las perspectivas: interseccional de género y de diversidad para garantizar de manera plena y efectiva los derechos de las mujeres en situación de discapacidad. Esto es, para abordar y revertir los obstáculos y riesgos particulares que enfrentan la diversidad de mujeres en situación de discapacidad, en función de cómo interactúan las distintas «identidades, condiciones y circunstancias vitales multidimensionales».

En consonancia con los instrumentos y estándares internacionales de derechos humanos, la Ley n. ${ }^{\circ} 19.580$ de Violencia hacia las Mujeres Basada en Género (Uruguay, 2017), define que

... la violencia basada en género es una forma de discriminación que afecta, directa o indirectamente, la vida, libertad, dignidad, integridad física, psicológica, sexual, económica o patrimonial, así como la seguridad personal de las mujeres.

Se entiende por violencia basada en género hacia las mujeres toda conducta, acción u omisión, en el ámbito público o el privado que, sustentada en una relación desigual de poder en base al género, tenga como objeto o resultado menoscabar o anular el reconocimiento, goce o ejercicio de los derechos humanos o las libertades fundamentales de las mujeres (art. 4).

La ley establece entre los principios que deben orientar su aplicación el Principio de igualdad y no discriminación, en función del cual prohíbe toda distinción, exclusión o restricción basada entre otros factores en la situación de discapacidad que tengan por objeto o resultado, el menoscabar o anular el reconocimiento, goce o ejercicio de los derechos humanos y las libertades fundamentales de las mujeres (Uruguay, 2017, art. 5).

Se desprende de la ley la necesidad de abordar la violencia basada en género y sus distintas formas, desde una perspectiva que tome en cuenta la interacción del género y la discapacidad.

Como sostiene Andrea Tuana (2020), la interseccionalidad se presenta indispensable para abordar la violencia basada en género abarcando su complejidad. En palabras de la autora:

La interseccionalidad propone, fundamentalmente, ir más allá de las consecuencias del patriarcado en las diversas formas de violencia, pues existen otras matrices de dominación, como son el heterosexismo, el racismo, el adultocentrismo, el clasismo, 
que tienen una injerencia interseccional en los procesos de exclusión y dominación que caracterizan a la violencia de género (p. 17).

\section{Participación y acceso a la información: dos factores fundamentales para los abordajes en torno a la discapacidad y los derechos humanos}

Finalmente, y con el propósito de terminar de enmarcar la experiencia de extensión que presentaremos en la tercera sección de este trabajo, nos interesa retomar y destacar dos aspectos que se desprenden del enfoque de derechos humanos de la discapacidad. Estos elementos se encuentran enfatizados en la CPDP sobre los Derechos de las Personas con Discapacidad sobre la cual ya nos hemos referido.

En tal sentido e incorporando la reivindicación histórica de los movimiento por los derechos de las personas en situación de discapacidad, el preámbulo de la CPDP enfatiza «que las personas con discapacidad deben tener la oportunidad de participar activamente en los procesos de adopción de decisiones sobre políticas y programas, incluidos los que les afectan directamente» (ONU, 2006, Preámbulo, lit. o). Asimismo, afirma la importancia de la accesibilidad a la información «para que las personas con discapacidad puedan gozar plenamente de todos los derechos humanos y las libertades fundamentales» (ONU, 2006, Preámbulo, lit. v). Estos aspectos son recogidos explícitamente en los artículos 4 y 9 de este instrumento.

En línea con el enfoque que se desprende de las citas anteriores el Área de Derechos Humanos ha venido trabajando considerando el acceso a la información como un eje transversal a sus abordajes.

Esta definición parte del convencimiento de que una de las notas fundamentales de la información, «es su carácter de medio o instrumento para el ejercicio de otros derechos» (Abramovich y Courtis, 2000). Bajo esta conceptualización el acceso a la información, además de ser un derecho humano, se torna una herramienta indispensable para que las personas puedan ejercer sus derechos y conocer los mecanismos para reclamarlos cuando estos no son garantizados, lo que cobra una importancia sustantiva en relación con los sectores históricamente excluidos o marginados de la sociedad (Comisión Interamericana de Derechos Humanos, 2012, párr. 5).

Este carácter instrumental del derecho de acceso a la información representa desafíos y obligaciones específicas para los Estados a efectos de garantizar su universalidad y plena vigencia bajo los principios de igualdad y no discriminación. La accesibilidad comunicacional constituye uno de los componentes del núcleo de tales retos y obligaciones, y no puede concebirse, sino como elemento sustantivo del derecho de acceso a la información.

Así, la Convención sobre los Derechos de las Personas con Discapacidad dispone que 
[a] fin de que las personas con discapacidad puedan vivir en forma independiente y participar plenamente en todos los aspectos de la vida, los Estados Partes deberán adoptar medidas pertinentes para asegurar el acceso de las personas con discapacidad, en igualdad de condiciones con las demás a la información (ONU, 2006, art. 9).

$\mathrm{Al}$ hacerlo, es fundamental que los Estados adopten el enfoque de la interseccionalidad.

En esa misma línea es importante considerar que puede negarse el acceso a la información no solo mediante la inexistencia de formatos accesibles, sino también sobre la base de estereotipos nocivos. Esto es especialmente recurrente en el caso de las mujeres en situación de discapacidad, que como ya fue planteado generalmente se encuentran con barreras actitudinales, prejuicios y preconceptos asociados a la discapacidad.

En palabras del CRPD:

Puede negarse a las mujeres con discapacidad el acceso a la información y la comunicación, incluida una educación sexual integral, sobre la base de estereotipos nocivos que suponen que son asexuales y, por tanto, no necesitan esa información en igualdad de condiciones con las demás (ONU, CRPD, 2016, párr. 40).

Precisamente bajo esta mirada interseccional, el Comité para la Eliminación de la Discriminación contra la Mujer (CEDAW, por sus siglas en inglés) ${ }^{7}$ recomendó al Estado uruguayo garantizar condiciones de accesibilidad de los servicios asociados a la protección y asistencia a situaciones de violencia basada en género (ONU, CEDAW, 2016, párr. 20, literal f) y la generación de datos específicos sobre la población de mujeres con discapacidad en diferentes ámbitos y con relación a situaciones de violencia basada en género (ONU, CEDAW, 2016, párr. 42).

Por su parte, el Comité sobre los Derechos de las Personas con Discapacidad recomendó al Estado uruguayo «que incluya a las mujeres con discapacidad en los planes y estrategias del Programa Nacional de Discapacidad (Pronadis) y del Instituto Nacional de las Mujeres (Inmujeres), revise las políticas sobre discapacidad para incluir un enfoque de género, y las de combate a la violencia contra las mujeres para incluir la dimensión de discapacidad» (ONU, CRPD, 2016, párr. 16).

La experiencia de extensión que el Área de Derechos Humanos viene desarrollando en torno a la articulación derechos humanos, discapacidad y género, responde a los parámetros conceptuales delineados en esta sección. Asimismo, retoma las observaciones finales realizadas a Uruguay por el CEDAW y el CRPD de la ONU.

7 El Comité para la Eliminación de la Discriminación contra la Mujer es el órgano de expertas independientes que supervisa la aplicación de la Convención sobre la Eliminación de todas las formas de Discriminación contra la Mujer. Los Estados parte de este tratado, se comprometen a presentar periódicamente informes al Comité en el que rinden cuentas sobre la implementación de los compromisos asumidos al ratificar este instrumento y las obligaciones derivadas para el Estado. El comité examina los informes y formula a cada Estado parte sus preocupaciones y recomendaciones en forma de observaciones finales. En 2016 el Comité adoptó sus observaciones finales sobre la aplicación de la Convención por parte del Estado uruguayo y emitió un conjunto de recomendaciones (ONU, CEDAW, 2016). 
Abordar desde la interseccionalidad el género y la discapacidad se traduce en la necesidad de desarrollar metodologías y repensar marcos teóricos para llevar a la práctica el enfoque de derechos humanos de la discapacidad en el quehacer universitario y la sociedad con miras a que sea un enfoque transversal a todo el hacer. Poner en común el camino recorrido por las distintas experiencias universitarias constituye un elemento indispensable para ese proceso. Tal es nuestro objetivo para la siguiente sección de este trabajo.

\section{Experiencia de trabajo con el Colectivo Mujeres y Discapacidad}

En setiembre de 2019 el equipo del Área Derechos Humanos inició su trabajo junto con el Colectivo Mujeres y Discapacidad, grupo formado en 2017 integrado por mujeres en situación de discapacidad y por mujeres sin discapacidad, nucleadas por la transversalización de género y discapacidad. Está conformado por mujeres que residen en los departamentos de Montevideo y Canelones. Este grupo cuenta con asistencia técnica de la Secretaría de Accesibilidad para la Inclusión de la Intendencia de Montevideo, quienes le proveen el servicio de intérprete de lengua de señas uruguaya (LSU) y un espacio de reunión (tanto presencial como virtual). La frecuencia de reunión del Colectivo es quincenal, con reuniones que van de dos a tres horas, contando con un robusto orden del día. El funcionamiento del espacio es horizontal, si bien se destacan algunos liderazgos predominantes, los roles rotan a la interna del colectivo. Tiene una metodología de trabajo pautada que incluye citaciones, orden del día, subgrupos de trabajo. El medio de comunicación privilegiado por el colectivo es el Whatsapp por sobre el correo electrónico.

En los inicios el trabajo desde el área estuvo centrado en acompañar las reuniones con escucha atenta a fin de delimitar la construcción de la demanda. Las primeras actividades que compartimos fueron las asociadas a la participación del colectivo en las movilizaciones del 8M. Hasta este momento el proceso de trabajo fue de cercanía, de encuentros que potenciaban y evidenciaban la diversidad constitutiva de este grupo de mujeres. Pero a partir del 13 de marzo del 2020 todo cambió.

Siguiendo los apuntes presentados por Agustín Cano y María Ingold (2020), la extensión fue una de las funciones universitarias que se vio más interpelada por las directrices de repliegue y confinamiento. Los procesos de trabajo extensionistas son por definición de proximidad, de articulación de saberes entre academia y sociedad. «En un escenario plagado de imposibles (no se puede salir, no se puede mantener encuentros presenciales, no se puede, no se puede), lo posible cobra otro valor» (Cano e Ingold, 2020, p. 40).

En marzo de 2020 y a partir de la situación de emergencia sanitaria y social desatada a raíz de la pandemia, el área implementó un relevamiento para identificar las demandas emergentes en el Colectivo Mujeres y Discapacidad. El relevamiento fue 
realizado a través de una consulta virtual que tuvo por objetivo identificar estas demandas y posibles líneas de trabajo en respuesta a ellas a promover desde la Udelar. La implementación de la consulta contó con el apoyo de integrantes del Colectivo, lo cual permitió fortalecer aspectos vinculados a su accesibilidad. Las preguntas incluidas fueron las siguientes: 1) Según tu experiencia personal ¿Cuáles son los principales problemas que enfrentan las personas en situación de discapacidad frente a la pandemia del coronavirus en Uruguay?; 2) ¿Cuáles son los problemas particulares que enfrentan las mujeres en situación de discapacidad en este contexto?; 3) ¿Cuáles te imaginas que podrían ser los aportes desde la Universidad para enfrentar estos problemas?; 4) Según tu conocimiento: ¿qué acciones sugerirías para minimizar las situaciones de violencia doméstica que podrían suceder hacia las mujeres en situación de discapacidad por la cuarentena?, y 5) ¿De qué manera estás informándote sobre el coronavirus en Uruguay?

El formulario estuvo activo 48 horas del 23 al 25 de marzo de 2020. Este tiempo estuvo acordado con las integrantes del colectivo. La vía de difusión del formulario a la interna del colectivo fue el grupo de Whatsapp y el apoyo de algunas integrantes del colectivo a sus compañeras para que pudieran completarlo resultó fundamental. En esta primera fase se contó con once respuestas. Desde el área se hizo una sistematización y análisis, las que pasaron a configurar distintas posibles líneas de acción.

Varias de las respuestas presentaron cruces temáticos asociados a actividades de la vida cotidiana que refieren a los cuidados, el acceso a la información y a situaciones de violencia. En función de los emergentes de este relevamiento el equipo universitario identificó la posibilidad de trabajar junto con el Colectivo Mujeres y Discapacidad en una acción que permitiera canalizar un conjunto de preocupaciones y propuestas existentes en este grupo: mejorar las políticas orientadas a abordar la situación violencia basada en género y visibilizar el efecto diferenciado y agravado de la pandemia sobre las mujeres en situación de discapacidad.

El trabajo junto con el colectivo permitió concretar un documento con aportes, insumos y propuestas de acciones concretas para fortalecer la respuesta estatal. Este fue formalmente presentado al Inmujeres en abril del mismo año.

Una mención especial en este artículo merece el proceso de construcción y la metodología de trabajo que cocreamos para concretar el documento de aportes en medio de imposibles. A modo de síntesis, la configuración del trabajo estuvo pautada por instancias de procesos en cadena, en la mayoría de los casos se recurrió a un trabajo subgrupal. Fue necesario adoptar distintas estrategias para presentar la información (video, audio, texto, LSU), contemplando criterios de accesibilidad para garantizar la participación de las mujeres del colectivo. Algunas de las mujeres que participaron de la construcción del petitorio cuentan con antecedentes en este tipo de activismo (por su profesión o trayectoria), mientras que para otras era su primera experiencia. Nuevamente, la diversidad aparece como un condimento definitorio del quehacer extensionista, desafiando y enriqueciendo la práctica. Asimismo, es importante 
mencionar que varias de las mujeres son adultas y adultas mayores por lo que no existían antecedentes de vinculación tecnológica, lo que por momentos complejizó la tarea.

En cuanto al documento en sí, este posibilitó al colectivo visibilizar algunos vacíos de la política pública y formular un conjunto propuestas concretas para la adopción de medidas que garanticen en la práctica y para todo el universo de mujeres, el acceso a la información y la accesibilidad de los servicios de asesoramiento y atención para mujeres en situación de violencia.

Entre otros puntos, el colectivo planteó la necesidad de que se adopten todas las medidas necesarias para garantizar la accesibilidad de la línea telefónica 08004141 (y de toda la información que permita acceder a este servicio) para mujeres sordas y mujeres en situación de discapacidad intelectual. Asimismo, destacó la necesidad de que se visibilice la situación de las mujeres en situación de discapacidad en el marco de las acciones de prevención de la violencia de género desarrolladas por el Estado en el contexto de la pandemia. En junio de 2020 el documento elaborado por el Colectivo Mujeres y Discapacidad también fue remitido al Pronadis del Ministerio de Desarrollo Social (MIDEs).

Al momento de finalizar la redacción de este artículo, y a casi un año de haber elevado el petitorio a las autoridades correspondientes, el Colectivo Mujeres y Discapacidad aún no cuenta con una respuesta formal del Inmujeres sobre las acciones planteadas y peticionadas. En el transcurso de los meses siguientes a la presentación del documento, el Colectivo solicitó y accedió a una reunión con el Pronadis para desarrollar lo planteado en el documento. En dicha reunión las autoridades adelantaron que distintas acciones de las solicitadas estaban contempladas en la proyección de medidas conjuntas entre el Pronadis e Inmujeres. Al cierre de este artículo, con el acompañamiento del Área de Derechos Humanos el colectivo se encontraba preparando una solicitud de acceso a la información pública en el marco de la Ley n.$^{\circ} 18.381$, a efectos de monitorear las medidas desarrolladas por el Inmujeres en relación con la atención de las situaciones de violencia basada en género para las mujeres en situación de discapacidad y las medidas planteadas por el Colectivo en su documento previo.

Esta segunda acción fue definida por el colectivo como parte de las acciones emprendidas en el marco de las movilizaciones y actividades desarrolladas en el contexto del $8 \mathrm{M}$ en reclamo de igualdad de género y del pleno reconocimiento de los derechos de las mujeres. De esta manera el Colectivo Mujeres y Discapacidad ha resuelto incorporar el uso de los mecanismos para el acceso a la información pública como herramienta de incidencia para continuar reivindicando y monitoreando el cumplimiento de las obligaciones del Estado de garantizar una vida libre de violencia para todas las mujeres. Frente a esta nueva acción concreta acordada por el colectivo y canalizada a través de un subgrupo de trabajo, el acompañamiento del área se ha orientado a: 1) cocrear con el subgrupo de trabajo una metodología que posibilite la preparación de la solicitud de información mediante intercambios virtuales, y 2) poner en común y a 
disposición del subgrupo redactor un conjunto de herramientas relacionadas con la regulación establecida por la Ley n. ${ }^{\circ} 18.381$, así como técnicas para la construcción de la demanda de información; 3 ) acompañar las reuniones del subgrupo de trabajo y el proceso de construcción de las preguntas que integrarán la solicitud de información.

Es importante mencionar que entre los temas que quedaron por fuera del petitorio, pero que se desprenden de las respuestas recibidas en el marco de la consulta instrumentada al inicio de la pandemia, están las que refieren al acceso a atención de la salud. Las integrantes del colectivo señalaron distintos niveles de imposibilidad o dificultad para poder mantener la atención de la salud derivada de acceso a vehículos para traslado o de la necesidad de prevención de circulación para mitigar el riesgo de contagio. También narraron distintas barreras comunicacionales para la atención de la salud de manera adecuada. Como ya hemos mencionado, el contexto de pandemia ha visibilizado y profundizado barreras ya existentes para la población en situación de discapacidad.

Desde nuestro hacer docente, entendemos que esta forma de trabajo que nos dimos con el colectivo en ese contexto tan desafiante responde a una concepción de universidad y de extensión latinoamericanas. Humberto Tommassino y Luis Barreras (2020) sintetizan este posicionamiento ético-profesional, reconociendo que la creación de estas prácticas de extensión dialogadas y gestadas en la diversidad contribuyen a la creación de «una universidad con sentido popular, sin distinción de claustros en la producción de ideas, que funden una nueva universidad, sin muros, abierta, grande» (Tommasino y Barreras, 2020, p. 16).

\section{Conclusiones}

En este artículo hemos reflexionado y argumentado en torno a las potencialidades y desafíos de la extensión universitaria para el trabajo en derechos humanos, género y discapacidad. Asimismo planteamos la vinculación de la extensión y la educación en derechos humanos y las oportunidades que este vínculo representa. Resulta difícil concluir cuando hay muchas líneas de trabajo aún abiertas con el Colectivo Mujeres y Discapacidad, pero asumimos el desafío.

Iniciamos estos apuntes finales destacando el rol fundamental que cumplió la Udelar en general, y la Extensión Universitaria en particular, en el contexto de pandemia. Universidad que tuvo la capacidad de desplegar sus acumulados y su creatividad para producir conocimiento y construir saberes situados de manera flexible y colaborativa. La vertiginosidad y la novedad hicieron que todas las decisiones y acciones debieran tomarse con celeridad. Sin embargo, esa celeridad mantuvo como constantes la apuesta a la construcción colectiva y a la reflexión crítica sobre las prácticas universitarias. En este proceso se produjeron algunas respuestas, pero sin dudas fueron más las interrogantes, las dudas y las potenciales líneas de trabajo que se abrieron a raíz de ese (este) contexto. 
Este tipo de actividades de extensión universitaria siguiendo los aportes de Boaventura de Sousa Santos (2010) pueden ser consideradas en clave de defensa de la diversidad cultural. Durante el desarrollo y presentación de la base conceptual y teórica que fundamenta nuestra práctica como docentes universitarias, se explicita que lo que nos hace iguales es el hecho de ser sujetos de derechos. Sin embargo, la diversidad cultural es inherente a la humanidad, razón por la cual debemos producir metodologías y diseños de intervención que la respeten, posibilitando el encuentro.

El proceso de cocreación del documento presentado ante el Inmujeres nos dejó como docentes muchos aprendizajes y desafíos en materia de accesibilidad comunicacional, y nos brindó nuevas herramientas y vivencias para una mejor comprensión de cómo operan las interseccionalidades en la vida cotidiana de las mujeres en situación de discapacidad.

Al mismo tiempo, mediante el proceso de elaboración de este documento el colectivo logró consensuar un diagnóstico sobre los obstáculos existentes para el acceso de las mujeres en situación de discapacidad a los servicios de asesoramiento sobre violencia doméstica y otras formas de violencia basada en género que brinda la línea telefónica o80o 4141. Asimismo, el propio colectivo llegó a acuerdos sobre las distintas acciones necesarias para revertirlos, y fundamentalmente un marco conceptual común a partir del cual posicionarse y reivindicar sus derechos. Entendemos también que el proceso llevado adelante contribuyó a que el colectivo pudiera ir encontrando nuevas formas de gestionar el encuentro, los consensos y disensos en un nuevo escenario pautado por el cambio de la presencialidad a la virtualidad.

Como ya se ha mencionado a lo largo de este artículo, promover procesos de extensión universitaria que tomen a la accesibilidad como una dimensión transversal, es un camino indispensable para seguir construyendo una Universidad comprometida con la igualdad y la no discriminación. Accesibilidad entendida en sus distintas dimensiones: la geográfica, la económica, en síntesis: la que posibilita que nadie quede afuera. Entendemos que la accesibilidad es sinónimo de habilitación, entendiéndose como contracara a la existencia de distintas barreras que imposibilitan la participación activa y el ejercicio de derechos de las mujeres en situación de discapacidad. Habilitar la construcción colectiva de procesos de denuncia, en los que las mujeres con discapacidad están directamente involucradas es condición necesaria para el empoderamiento de este colectivo. El movimiento social de la discapacidad, que tiene su origen en las décadas del sesenta y del setenta, cuando los distintos colectivos de personas con discapacidad ocupan el espacio público en Estado Unidos y en el Reino Unido en reclamo de sus derechos, tiene como máxima expresión «Nada sobre nosotros sin nosotros». Esta máxima atraviesa las prácticas extensionistas que se impulsan desde el Área de Derechos Humanos, construyendo en permanente sinergia con los propios colectivos.

Estamos en tiempos de revolución, revolución tecnológica, revolución de información, revoluciones políticas, en tal sentido concluimos este artículo tomando 
las reflexiones sobre las universidades latinoamericanas de Tommassino y Barreras (2020), quienes, además de otorgarles identidad popular, las comprometen al aporte de la revolución epistemológica. Podríamos aventurarnos a concluir que estamos en tiempos de revolución epistemológica, de revisión de las prácticas a velocidades que desafían el ejercicio docente. Nuestra invitación es a que dicha revolución no deje a nadie afuera, que sea una revolución desde el encuentro y la proximidad que tiene la práctica extensionista, aun cuando dicho encuentro está mediado por las pantallas.

\section{Referencias}

Abramovich, V., y Courtis, Ch. (2000). El acceso a la información como derecho. En E. L. Duhalde, Anuario de Derecho a la Comunicación, 1(1). Buenos Aires: Siglo Veintiuno Editores. Recuperado de https://www.cels.org.ar/common/documentos/acceso_informacion_como_derecho.pdf

Bordoli, E. (2010). Aportes para pensar la extensión universitaria. En J. C. CARrasco, R. Cassina y H. Tommasino (Eds.), Extensión en obra. Experiencias, reflexiones, metodologías y abordajes en extensión universitaria (pp. 13-20). Montevideo: Universidad de la República.

Cano, A., e Ingold, M. (2020). La extensión universitaria en tiempos de pandemia: lo que emerge de la emergencia. Redes de Extensión (7), 38-45. Recuperado de: http://revistascientificas.filo.uba.ar/ index.php/redes/article/view/9169/7956

Comisión Interamericana de Derechos Humanos (2012). El derecho de acceso a la información en el marco jurídico interamericano. Recuperado de https://www.oas.org/es/cidh/expresion/docs/ publicaciones/acceso\%20a\%2ola\%2oinformacion\%202012\%202da\%2oedicion.pdf.

CubiLlos, J. (2015). La importancia de la interseccionalidad para la investigación feminista. Oxímora Revista Internacional de Ética y Política, 7, 119-137. Recuperado de https://revistes.ub.edu/index. php/oximora/article/view/14502/17834.

De Sousa SAnTOS, B. (2010). La Universidad en el siglo XXI, para una reforma democrática y emancipatoria de la Universidad. La Paz: Plural.

Mujica, R. (2002). La metodología de la educación en derechos humanos. Revista Instituto Interamericano de Derechos Humanos, 36, 341-364. Recuperado de https://www.corteidh.or.cr/tablas/Ro6835-13. pdf

ORganización De Naciones Unidas (ONU) (2006). Convención sobre los Derechos de las Personas con Discapacidad [en línea]. Recuperado de: https://www.un.org/esa/socdev/enable/documents/ tccconvs.pdf.

ONU (2011). Declaración de las Naciones Unidas sobre educación y formación en materia de derechos humanos. [en línea]. Recuperado de: https://www.ohchr.org/sp/issues/education/educationtraining/ pages/undhreducationtraining.aspx.aspx

ONU, CRPD (2016). Observaciones finales sobre el informe inicial del Uruguay. Recuperado de https://www.cainfo.org.uy/2016/o9/recomendaciones-a-uruguay-del-comite-sobre-los-derechos-de-las-personas-con-discapacidad-de-naciones-unidas/

ONU, CEDAW (1979). Convención sobre la eliminación de todas las formas de discriminación contra la mujer. Recuperado de: https://www.ohchr.org/sp/professionalinterest/pages/cedaw.aspx

ONU, CEDAW (2016). Observaciones finales sobre los informes periódicos octavo y noveno combinados del Uruguay. Recuperado de https://tbinternet.ohchr.org/_layouts/15/treatybodyexternal/ Download.aspx?symbolno=CEDAW/C/URY/CO/8-9\&Lang $=\mathrm{Sp}$ 
Rodino, A. (2014). Pensar la educación en derechos humanos como política pública. Revista de Ciencias Sociales, segunda época, (25), 129-139. http://www.unq.edu.ar/advf/documentos/5939558c6ba3b.pdf

Servicio Central de Extensión y Actividades en el Medio (2019). Área Derechos Humanos [Sitio web]. Montevideo: Universidad de la República. Recuperado de https://www.extension.udelar. edu.uy/derechos-humanos-ddhh/

Tommasino, H., y Barreras, L. (2020). Extensión crítica e integralidad en tiempos de pandemia: sus aportes para una Universidad Latinoamericana en la encrucijada. [Manuscrito inédito].

Tuana, A. (2020). Violencia de género. Discursos patriarcales restauradores de la subordinación de las mujeres. En Red Uruguaya contra la Violencia Doméstica y Sexual, Miradas sobre violencia basada en género y generaciones. Montevideo: RUCVDS. Recuperado de http://www.violenciadomestica.org.uy/repo/img/miradassobreviolenciabasadaengneroygeneracionesvbgg.pdf.

UniversidAd de LA República (1958, octubre 29). Ley n. ${ }^{0}$ 12.549: Ley Orgánica de la Universidad de la República. Recuperado de: https://dgjuridica.udelar.edu.uy/ley-organica/.

URUguay (2008, noviembre 20). Ley n. ${ }^{\circ}$ 18.418: Convención de Naciones Unidas sobre los Derechos de las Personas con Discapacidad. Recuperado de http://www.impo.com.uy/bases/leyes/18418-2008.

Uruguay (2017, diciembre 22). Ley n. ${ }^{\circ}$ 19.580: Ley de Violencia hacia las Mujeres Basada en Género. Recuperado de https://www.impo.com.uy/bases/leyes/19580-2017.

Willat, F. (2011). ¿Qué es la perspectiva de derechos humanos? Hablando de derechos $\mid$ DESC+A Charlas de formación en derechos humanos, (2). Montevideo: Ministerio de Desarrollo Social. Recuperado de http://dspace.mides.gub.uy:808o/xmlui/bitstream/handle/123456789/1842/DESC\%2BA\%2O -\%2001\%20-\%20Qu\%C3\%A9\%20es\%2ola\%2operspectiva\%2oen\%2oderechos\%2ohumanos. pdf? sequence $=1 \&$ isAllowed $=y$. 